\title{
Multifactorial Assessment of Risk of Falling in 753 Post-Menopausal Women: A Multicenter Cross-Sectional Study by the Italian Group for the Study of Metabolic Bone Diseases
}

This article was published in the following Dove Press journal:

Clinical Interventions in Aging

Giovanni lolascon, (D) Alessandro de Sire, (D) ${ }^{1-3}$ Dario Calafiore, (D) ${ }^{1,4}$ Maria Grazia Benedetti, ${ }^{5}$ Carlo Cisari, ${ }^{2,6}$ Giulia Letizia Mauro, ${ }^{7}$ Silvia Migliaccio, ${ }^{8}{ }^{8}$ Ranuccio Nuti, ${ }^{9}$ Giuseppina Resmini, ${ }^{10}$ Stefano Gonnelli, ${ }^{9}$ Antimo Moretti (iD)

'Department of Medical and Surgical Specialties and Dentistry, University of Campania "Luigi Vanvitelli”, Naples, Italy;

${ }^{2}$ Physical and Rehabilitative Medicine, Department of Health Sciences, University of Eastern Piedmont "A. Avogadro", Novara, Italy; ${ }^{3}$ Rehabilitation Unit, "Mons L. Novarese" Hospital, Vercelli, Moncrivello, Italy; ${ }^{4}$ Section of Neuromotor

Rehabilitation, Department of Neuroscience, ASST Carlo Poma, Mantova, Italy; ${ }^{5}$ Physical Medicine and Rehabilitation Unit, IRCCS-Istituto Ortopedico Rizzoli, Bologna, Italy; ${ }^{6}$ Physical Medicine and Rehabilitation Unit, University Hospital "Maggiore della Carità", Novara, Italy; ${ }^{7}$ Department of Surgical and Oncology Sciences, University of Palermo, Palermo, Italy; ${ }^{8}$ Department of Movement, Human and Health Sciences, Section of Health Sciences, University of Rome, Foro Italico, Rome, Italy; 'Department of Medicine, Surgery and Neuroscience, University of Siena, Siena, Italy; ${ }^{10}$ Section of Orthopaedics and Traumatology, Treviglio-Caravaggio Hospital, Treviglio, Bergamo, Italy

Correspondence: Alessandro de Sire Physical and Rehabilitative Medicine, Department of Health Sciences, University of Eastern Piedmont "A. Avogadro", Viale Piazza D’Armi I, Novara 28I00, Italy Tel +3903213734800

Email alessandro.desire@gmail.com
Objective: To assess physical performance, number of falls, previous fragility fractures, and ongoing pharmacological therapy in a cohort of post-menopausal women, according to their risk of falling.

Methods: In this multicenter cross-sectional study, we recruited in a 3-year period (May 2016 to April 2019), women aged >60 years referred to seven Osteoporosis and Bone Metabolism Outpatient Services of the Italian Group for the Study of Metabolic Bone Diseases. The study population was divided into three groups according to the risk of falling, assessed through the Elderly Fall Screening Test (EFST): low risk (EFST score $=0$ 1); moderate risk $(E F S T=2-3)$; high risk $(E F S T=4-5)$. Outcome measures were: 4-meter gait speed (4MGS); unipedal stance time (UST); number of falls in the previous year; previous fragility fractures; ongoing pharmacological therapy.

Results: We analyzed 753 women (mean aged 70.1 \pm 9.2 years): $378(50.2 \%)$ at low risk of falling, $247(32.8 \%)$ at moderate risk, and $128(17.0 \%)$ at high risk. 4MGS and UST resulted as pathological in the $93.9 \%$ and $99.2 \%$, respectively, of women at high risk. There were significant differences among groups for both outcomes $(p<0.001)$. There was also a significant difference among groups $(p<0.001)$ in terms of previous falls and fragility fractures. Lastly, there were significant differences $(p<0.05)$ among groups in using antihypertensive drugs, antiplatelet agents, anticoagulants, antidepressants, anti-osteoporotic drugs, and vitamin $\mathrm{D}$, and/or calcium supplementation.

Conclusion: Physical performance, prevalence of falls and fragility fractures, and an assessment of pharmacological therapy should be investigated in post-menopausal women because of their significant correlation with risk of falling.

Keywords: accidental falls, osteoporosis, walking speed, fractures, bone, rehabilitation

\section{Introduction}

A fall is defined by the World Health Organization as "inadvertently coming to rest on the ground, floor or other lower level." Although this event occurs accidentally, several risk factors have been identified. ${ }^{2-4}$ Over a third of older people fall each year with unfavorable clinical consequences and a significant socio-economic burden. ${ }^{5}$ Falls, indeed, are responsible for over $13 \%$ of ambulance interventions in the emergency medical services, ${ }^{6}$ and for over 3 million hospitalizations, with a total cost exceeding 25 billion euros per year in Europe, most of which concern osteoporosis fractures. ${ }^{6-9}$ 
The assessment of fall risk in the elderly is considered as a cornerstone for the prevention of fragility fractures. There are several validated tools to provide this approach in older non-institutionalized people, such as the Performance Oriented Mobility Assessment or Tinetti Test, ${ }^{10}$ and the Timed Up and Go Test. ${ }^{11}$ In 1998, Cwikel et al proposed an easy administration and understanding test, the Elderly Fall Screening Test (EFST), to stratify the risk of falling in community-dwelling elderly. ${ }^{12}$ EFST items include both patient-reported events and functional evaluations performed by an examiner. In contrast to other assessment tools, it also takes into account two significant details, such as the number of falls and "near falls" that occurred during the previous year. "Near fall" is a stumble event or loss of balance that would result in a fall if sufficient recovery mechanisms were not activated. ${ }^{13}$ It has been recently highlighted that adults who experienced "near falls," which are more prevalent than effective falls, ${ }^{14}$ present a greater risk of incident falls. ${ }^{15}$

Postural instability and impaired gait pattern result in an increased risk of falling and consequently fractures, and are frequently associated with sarcopenia, ${ }^{16}$ a condition commonly defined as a reduction in muscle mass combined with a decline in muscle function ${ }^{17}$ with a high prevalence not only in older people but also in the younger population, including post-menopausal women.

To date, several studies have investigated the association between physical performance and risk of falling in older women, ${ }^{18-21}$ although without a wide assessment of all the impairments that characterize the aging population. Therefore, in the present study, we sought to evaluate physical performance according to the risk of falling in a cohort of community-dwelling post-menopausal women.

\section{Methods}

\section{Participants}

In this multicenter cross-sectional study, we recruited in a 3-year period (May 2016 to April 2019) communitydwelling post-menopausal women aged $>60$ years referred to seven Osteoporosis and Bone Metabolism Outpatient Services of the Italian Group for the Study of Metabolic Bone Diseases (Gruppo Italiano di Studio delle Malattie del Metabolismo Osseo, GISMO): University Hospital "Maggiore della Carità" in Novara, Hospital "TreviglioCaravaggio" in Treviglio (BG), Rizzoli Orthopedic Institute in Bologna, University Hospital "Le Scotte" in Siena, University Hospital Umberto I in Rome, University
Hospital "Luigi Vanvitelli" in Napoli, and University Hospital "Paolo Giaccone" in Palermo. Exclusion criteria were: a) history of neuromuscular disease; b) cognitive impairment; c) hip or knee surgery in the previous 6 months; d) recent traumas; e) need of assistance in performing activities of daily living (ADL); f) walking aids use; g) severe knee and/or hip osteoarthritis (grade IV according to the Kellgren \& Lawrence classification). ${ }^{22}$

The study protocol was approved by the Institutional Review Boards and met the guidelines of the responsible governmental agency. At enrollment, all the participants were asked to carefully read and sign an informed consent, and researchers provided to protect the privacy, and the procedures in this study were in accordance with the Declaration of Helsinki, with pertinent national and international regulatory requirements. All participants were free to withdraw from the study at any time.

\section{Outcome Measures}

In the entire cohort, we assessed the risk of falling through EFST, ${ }^{12}$ consisting of 5 items with dichotomous variables (range $0-1$ ), with a total score ranging from 0 (lower risk of falling) to 5 (higher risk of falling). The first 3 items investigate potential falls in the previous year, any traumatic lesion reported (ie, injury, abrasion, hematoma, fracture) because of falling and the frequency of "near falls." The fourth item is a walking speed test which assesses the ability to walk over a 5-meter distance on a flat surface in 10 seconds. The last item evaluates the gait pattern, differentiating it into straight and safe or shuffling and unsteady gait. According to the EFST score, study participants have been divided into 3 groups at different risk of falling: low (EFST 0-1), moderate (EFST 2-3), or high risk (EFST 4-5).

Outcome measures of this cross-sectional study were:

(a) gait speed, evaluated by 4-meter gait speed (4MGS) with a cutoff value for poor physical performance of $\leq 0.8 \mathrm{~m} / \mathrm{s},{ }^{23}$

(b) balance, assessed by the unipedal stance test (UST), consisting of asking the patient to maintain a static open-eyed mono-podalic position (on the dominant lower limb) with arms crossed on the chest, starting from the moment the contralateral foot was raised from the ground; the test was considered as concluded if the subject: 1) used upper limbs to support herself; 2) moved the raised limb or touched the ground with it; 3) moved the weight-bearing leg to keep the balance; 4) maintained balance over 45 seconds; this 
task was repeated for three times and the best time was recorded. UST score was considered as pathological if it exceeded the cutoff by gender and age, with open eyes, proposed by Springer et al; ${ }^{24}$

(c) number of falls in the previous year, stratified into 3 categories (no falls, 1 fall, or recurrent falls if $\geq 2$ );

(d) number of vertebral fragility fractures;

(e) number of hip fragility fractures;

(f) number of non-vertebral and non-hip (NVNH) fragility fractures;

(g) ongoing pharmacological therapy (corticosteroids, nonsteroidal anti-inflammatory drugs, opioids, antihypertensive drugs, anti-arrhythmic drugs, antiplatelet agents, anticoagulants, statins, anticonvulsants, antidepressants, immunosuppressants, chemotherapeutics, L-thyroxine, aromatase inhibitors, anti-anxiety drugs/sedative-hypnotics and other treatments), anti-osteoporotic drugs, and vitamin D and/or calcium supplementation.

\section{Statistical Analysis}

Data management and analyses were conducted according to a pre-specified statistical analytical plan. Statistical analysis was performed using STATA v.12 (StataCorp LP, College Station, TX). The continuous variables are presented as means \pm standard deviations, whereas categorical data are expressed as counts (percentages). We performed the Shapiro-Wilk normality test, and because the data did not follow a normal distribution, we used Pearson's
Chi-squared test to assess differences among categorical data and the Kruskal-Wallis test to assess differences among continuous data. Moreover, the Spearman coefficient of correlation was used to test the correlation between EFST and 4MGS and UST. All the statistical tests were carried out on a two-sided significance level of 0.05 .

\section{Results}

In this multicenter cross-sectional study, we included 753 women, mean aged $70.1 \pm 9.2$ years, with a mean body mass index of $26.6 \pm 4.7 \mathrm{~kg} / \mathrm{m}^{2}$. According to the EFST score, the study participants have been divided into 3 groups: $378(50.2 \%)$ at low risk of falling (EFST 0-1), $247(32.8 \%)$ at moderate risk (EFST 2-3), and 128 $(17.0 \%)$ at high risk (EFST 4-5).

We found statistically significant differences in all outcome measures among the three groups (see Table 1 for further details). A reduced $4 \mathrm{MGS}(\leq 0.8 \mathrm{~m} / \mathrm{s})$ was found in $40.2 \%$ of women with EFST $0-1$, in $69.6 \%$ of women at moderate risk of falling, and in $93.9 \%$ of women at high risk of falling, with a significant difference among groups $(p<0.001)$. According to the cutoffs by gender and age, UST resulted as pathological in $76.7 \%$ of women at low risk of falling, in $90.7 \%$ of women at moderate risk, and in almost all (99.2\%) women at high risk, with a statistically significant difference among groups $(p<0.001)$. There were also significant differences among groups $(p<0.001)$ in the number of vertebral, hip, and NVNH fragility fractures, with a higher prevalence in the group at high risk of falling (as shown in Table 1).

Table I Differences in Outcome Measures Among Groups in the Study Population $(n=753)$

\begin{tabular}{|c|c|c|c|c|}
\hline & $\begin{array}{l}\text { At Low Risk of Falling } \\
(E F S T=0-1) \\
(n=378)\end{array}$ & $\begin{array}{l}\text { At Moderate Risk of Falling } \\
(E F S T=2-3) \\
(n=247)\end{array}$ & $\begin{array}{l}\text { At High Risk of Falling } \\
(E F S T=4-5) \\
(n=\mid 28)\end{array}$ & $P$-values \\
\hline 4MGS (m/s) & $0.89 \pm 0.34$ & $0.57 \pm 0.38$ & $0.37 \pm 0.20$ & $<0.001 * *$ \\
\hline $4 M G S \leq 0.8 \mathrm{~m} / \mathrm{s}$ & $152(40.21)$ & $172(69.64)$ & $120(93.75)$ & $<0.00 I^{*}$ \\
\hline UST (s) & $15.70 \pm 15.04$ & $8.39 \pm 10.37$ & $3.17 \pm 2.87$ & $<0.001 * *$ \\
\hline Pathological UST & $290(76.72)$ & $224(90.69)$ & 127 (99.22) & $<0.00 I^{*}$ \\
\hline \multicolumn{5}{|l|}{ Falls in the previous year } \\
\hline 0 & $322(85.19)$ & $58(23.48)$ & $0(0.00)$ & $<0.00 I^{*}$ \\
\hline I & $52(13.76)$ & $107(43.32)$ & $61(47.66)$ & $<0.00 I^{*}$ \\
\hline$\geq 2$ & $4(1.06)$ & $82(33.20)$ & $67(52.34)$ & $<0.00 I^{*}$ \\
\hline Vertebral fragility fractures & 42 (II.II) & $65(26.32)$ & $67(52.34)$ & $<0.001 *$ \\
\hline Hip fragility fractures & $6(1.59)$ & $25(10.12)$ & $36(28.13)$ & $<0.00 I^{*}$ \\
\hline NVNH fractures & $19(5.03)$ & $14(5.67)$ & $14(10.94)$ & $<0.00 I^{*}$ \\
\hline
\end{tabular}

Notes: Values are expressed as mean \pm standard deviation for continuous data and counts (percentages) for categorical data. $P$-values are considered significant when $P$ is less than 0.05. *Pearson's Chi-squared test; **Kruskal-Wallis test.

Abbreviations: EFST, Elderly Fall Screening Test; 4MGS, 4-meter gait speed; UST, unipedal stance test; NVNH, non-vertebral and non-hip. 
Furthermore, significant moderate negative correlations were observed for both comparisons between EFST and gait speed $(\rho=-0.575 ; p<0.001)$ and between EFST and UST $(\rho=-0.527 ; p<0.001)$.

In the study population, 667 patients $(88.6 \%)$ were receiving pharmacological therapies, as shown in Table 2. According to the EFST score, there were statistically significant differences among groups in terms of antihypertensive drugs $(p<0.001)$, antiplatelet agents $(p=0.002)$, anticoagulants $(p<0.001)$, and antidepressants $(p=0.017)$. Lastly, we reported that the post-menopausal women at high risk of falling (EFST 4-5) showed a significantly higher consumption of anti-osteoporotic drugs, and vitamin $\mathrm{D}$ and/or calcium supplementation than the other groups $(p=0.001)$.

\section{Discussion}

The results of this multicenter cross-sectional study suggest that community-dwelling post-menopausal women at high risk of falling have the worst physical performance in terms of gait speed and balance.

These findings are in line with those reported in other studies in the literature. ${ }^{25-27}$ Shimada et al showed that fallers had significantly lower gait speed compared to nonfallers, suggesting that a slow gait was associated with an increased risk of falls. ${ }^{25}$ Moreover, it has been demonstrated that poor physical performance has good accuracy for predicting falls and consequent hospitalization. ${ }^{26,27}$

In our population, the history of falls in patients with EFST high score was more prevalent than in the other two groups. Furthermore, it should be highlighted the high prevalence of fragility fractures (vertebral, hip, and $\mathrm{NVNH}$ ) in women at high risk of falling. In particular, in this group, more than half of patients had at least one vertebral fragility fracture.

Our data suggest that the higher the risk of falling, the worse were gait speed and balance in post-menopausal women, as demonstrated by the moderate correlation

Table 2 Differences in Pharmacological Therapies Among Groups in Patients Undergoing Any Treatment $(n=667)$

\begin{tabular}{|c|c|c|c|c|}
\hline & $\begin{array}{l}\text { At Low Risk of } \\
\text { Falling } \\
(\text { EFST }=0-1) \\
(n=3 \mid 8)\end{array}$ & $\begin{array}{l}\text { At Moderate Risk of } \\
\text { Falling } \\
(E F S T=2-3) \\
(n=225)\end{array}$ & $\begin{array}{l}\text { At High Risk of } \\
\text { Falling } \\
(\text { EFST }=4-5) \\
(n=124)\end{array}$ & $P$-values \\
\hline Corticosteroids & $21(6.6)$ & $23(10.2)$ & $12(9.7)$ & 0.271 \\
\hline NSAIDs & $4 I(12.9)$ & $42(18.7)$ & $25(20.2)$ & 0.077 \\
\hline Opioids & $2(0.6)$ & $3(1.3)$ & $2(1.6)$ & 0.550 \\
\hline Antihypertensive drugs & $172(54.1)$ & $149(66.2)$ & $94(75.8)$ & $<0.001$ \\
\hline Anti-arrhythmics & $14(4.4)$ & $20(8.9)$ & $8(6.5)$ & 0.107 \\
\hline Antiplatelet agents & $54(17.0)$ & $65(28.9)$ & $34(27.4)$ & 0.002 \\
\hline Anticoagulants & $15(4.7)$ & $16(7.1)$ & $27(21.8)$ & $<0.001$ \\
\hline Statins & $79(24.8)$ & $60(26.7)$ & $26(21.0)$ & 0.507 \\
\hline Antiepileptics & $5(1.6)$ & $7(3.1)$ & $4(3.2)$ & 0.332 \\
\hline Antidepressants & $16(5.0)$ & $19(8.4)$ & $16(12.9)$ & 0.017 \\
\hline Immunosuppressants & $5(1.6)$ & $2(0.9)$ & $3(2.4)$ & 0.530 \\
\hline Chemotherapeutics & I $(0.3)$ & I (0.4) & $\mathrm{I}(0.8)$ & 0.770 \\
\hline L-thyroxine & $60(18.9)$ & $40(17.8)$ & $24(19.4)$ & 0.922 \\
\hline Aromatase inhibitors & $6(1.9)$ & $6(2.7)$ & $3(2.4)$ & 0.783 \\
\hline Anti-anxiety/sedative-hypnotics & $21(6.6)$ & $25(11.1)$ & $9(7.3)$ & 0.167 \\
\hline \multicolumn{5}{|c|}{$\begin{array}{l}\text { Anti-osteoporotic drugs and/or vitamin D and/or calcium } \\
\text { supplementation }\end{array}$} \\
\hline Bisphosphonates & $72(22.6)$ & 78 (34.7) & $58(46.8)$ & 0.001 \\
\hline Denosumab & $10(3.1)$ & $27(12.0)$ & $21(16.9)$ & 0.001 \\
\hline Teriparatide & $3(0.9)$ & $5(2.2)$ & II (8.9) & 0.001 \\
\hline Vitamin D supplementation & $140(44.0)$ & $87(38.7)$ & $86(69.4)$ & 0.001 \\
\hline Calcium supplementation & $39(12.3)$ & $22(9.8)$ & $40(32.3)$ & 0.001 \\
\hline
\end{tabular}

Notes: Values are expressed as mean \pm standard deviation for continuous data and counts (percentages) for categorical data. $P$-values are considered significant when $P$ is less than 0.05 . The Pearson's Chi-squared test was performed.

Abbreviations: EFST, Elderly Fall Screening Test; NSAIDs, nonsteroidal anti-inflammatory drugs. 
between EFST score and both gait speed and UST. Taking into account that major risk factors for falls are identified as impairments of balance and gait, polypharmacy, history of falls and "near falls," EFST might be considered as a reliable tool to detect individuals at high risk of falling; our data support its role in this context, also considering that it is easy to use and quick to perform.

It might be easily hypothesized that a worse physical performance is a determining factor for falls and consequent fragility fractures. All strategies aimed at improving physical performance might significantly prevent the occurrence of these disabling conditions. In our sample, as we could expect, the women at higher risk of falling had a significantly higher number of osteoporotic fractures and a worse physical performance. Taken together, these findings confirm that osteoporosis and muscle wasting could go hand-in-hand in the elderly. ${ }^{28}$ The concept of "osteosarcopenia" describes a subset of older persons affected by a combination of low bone density (osteopenia/osteoporosis) and low muscle mass, strength, and/or functional capacity (sarcopenia). ${ }^{29-31}$ To manage this pathological condition, it is mandatory to diagnose it through adequate assessment of muscle function, particularly measuring gait speed. ${ }^{31}$ Moreover, Bhasin et $\mathrm{al}^{32}$ have recently shown that low gait speed might independently predict falls in community-dwelling older adults. Our study also confirms the role of this outcome measure as a quick, safe, and highly reliable test for physical performance. ${ }^{33}$ It has been demonstrated that gait speed might predict several adverse outcomes, such as falls, disability, need for institutionalization, and mortality. ${ }^{34-36}$

From our data analysis, it is not surprising that women at high risk of falling consumed more antihypertensive drugs $(p<0.001)$, antiplatelet agents $(p=0.002)$, anticoagulants $(p<0.001)$, and antidepressants ( $p=0.017)$ than the others. These findings are intriguing and might lead to several considerations on the relationship between polypharmacotherapy and risk of falling. Moreover, we can confirm that our results are in line with a recent longitudinal study performed by Dhalwani et $\mathrm{al}^{37}$ that showed that almost one-third of the population using five or more drugs had a significantly higher rate of falls over a 2-year period. It should be underlined that in our cohort, the $12.9 \%$ of women at high risk of falling were taking antidepressants, which are considered as the psychotropic drugs most strongly related to fall injuries. ${ }^{38}$ However, the link between drugs associated with increased risk of falling and any of the parameters of physical performance, such as gait speed and balance, deserves to be further investigated in future studies.

Lastly, we reported that the post-menopausal women at high fall risk (EFST 4-5) were receiving more antiosteoporotic drugs, vitamin D, and/or calcium supplementation than the other groups $(p=0.001)$, presumably due to the higher percentage of fragility fractures. Our study group has previously demonstrated the correlation between vitamin $\mathrm{D}$ deficiency and poor muscle function, ${ }^{21,39-41}$ but it is still debated if vitamin D supplementation could reduce the percentage of fallers. ${ }^{42}$ Further prospective studies are required to better elucidate the role of vitamin $\mathrm{D}$ in this context.

There is no doubt that the prevention of falls is better and more cost-effective than trying to address their consequences, such as fragility fractures. ${ }^{7,43}$ Therefore, starting from an accurate definition of key risk factors, rehabilitation might have a main role in the prevention of falls. According to this management strategy, the Centers for Disease Control and Prevention (CDC) ${ }^{44}$ have recently developed an algorithm to provide a multifactorial intervention for the elderly, according to specific risk factors and the number of falls. Moreover, the National Institute for Health and Care Excellence Guidelines ${ }^{45}$ recommended strengthening and balance training exercises for the elderly, considering that these interventions have been shown to significantly reduce the rate of falls. Indeed, in older people, particularly those with a history of previous fragility fractures, it is mandatory to implement an adequate individual rehabilitation project, including therapeutic exercise (early mobilization, structured exercise programs, strengthening, and balance training) for improving functional recovery and reducing the risk of falls and other fractures. ${ }^{46}$ Therapeutic exercise interventions have highly site-specific effects on bone tissue, and mainly forceful, rapid movements that load bones from many directions are known as the most beneficial; ${ }^{47}$ indeed, repetitive movements or low-impact exercises achievable in the elderly might prevent new incident fragility fractures. ${ }^{47}$

Even if it is difficult to perform a high-impact activity in older people because of their limited physical capacity or their comorbidities, the Position Paper of the Italian Society of Orthopaedics and Medicine ${ }^{49}$ has clearly defined specific treatment strategies for sarcopenic older people to perform safe, feasible, and effective aerobic, resistance, and power training exercises.

Moreover, it is also strongly recommended to treat agerelated muscle loss through the prescription of resistance- 
based physical activity, and conditionally recommended an adequate protein supplementation. ${ }^{50-59}$ Indeed,the role of a protein-rich diet and adequate use of dietary supplements in post-menopausal women and generally in older people has to be highlighted. ${ }^{51-55}$ A combination of physical exercise and amino acid supplementation might prevent or contrast muscle wasting in the elderly, as already shown by several studies. ${ }^{56-59}$

\section{Study Limitations}

This study has some limitations that have to be mentioned: firstly, the cross-sectional design of the study prevents a causal relationship from being established between low physical performance and falling rate; secondly, lack of data on muscle strength, a parameter that might be useful to better define fall risk in our cohort; thirdly, absence of data on the level of physical activity and on the nutritional assessment in our cohort (dietary intake, amino acid supplementation, use of dietary supplements, etc.); lastly, the absence of biochemical assessment of bone metabolism.

\section{Conclusion}

The findings of this multicenter cross-sectional study suggested that community-dwelling post-menopausal women at high risk of falling had worse physical performance, a higher prevalence of previous falls and osteoporotic fractures, and consumed more drugs. Therefore, it is mandatory to promote the prevention of falls in older people by developing a specific multidisciplinary community program. Outcome measures addressing physical function should be routinely provided in both primary care and rehabilitation settings in order to assess the risk of falling and to provide a patient-tailored multifactorial treatment.

\section{Funding}

There is no funding to report.

\section{Disclosure}

The authors report no conflicts of interest in this work.

\section{References}

1. World Health Organization. WHO global report on falls prevention in older age. Geneva: World Health Organization; 2008.

2. Deandrea S, Lucenteforte E, Bravi F, Foschi R, La Vecchia C, Negri E. Risk factors for falls in community-dwelling older people: a systematic review and meta-analysis. Epidemiology. 2010;21 (5):658-668. doi:10.1097/EDE.0b013e3181e89905

3. Nitz JC, Stock L, Khan A. Health-related predictors of falls and fractures in women over 40. Osteoporos Int. 2013;24(2):613-621. doi:10.1007/s00198-012-2004-z
4. Ambrose AF, Cruz L, Paul G. Falls and fractures: a systematic approach to screening and prevention. Maturitas. 2015;82(1):85-93. doi:10.1016/j.maturitas.2015.06.035

5. Tinetti ME, Kumar C. The patient who falls: "it's always a trade-off". JAMA. 2010;303(3):258-266. doi:10.1001/jama.2009.2024

6. Zduńczyk Ł, Leszczyński PK, Detsyk O, Charuta A. Analgesia in trauma patients administered by Emergency Medical Services. Crit Care Innov. 2019;2(1):11-21.

7. Gillespie LD, Robertson MC, Gillespie WJ, et al. Interventions for preventing falls in older people living in the community. Cochrane Database Syst Rev. 2009;15(2):CD007146.

8. Heinrich S, Rapp K, Rissmann U, Becker C, Konig HH. Cost of falls in old age: a systematic review. Osteoporos Int. 2010;21:891-902. doi:10.1007/s00198-009-1100-1

9. Lopez-Torres Hidalgo J, Group A. Prevention of falls and fractures in old people by administration of calcium and vitamin D. Randomized clinical trial. BMC Public Health. 2011;11(1):910. doi:10.1186/14712458-11-910

10. Tinetti ME, Williams TF, Mayewski R. Fall risk index for elderly patients based on number of chronic disabilities. Am J Med. 1986;80 (3):429-434. doi:10.1016/0002-9343(86)90717-5

11. Podsiadlo D, Richardson S. The timed "up \& go": a test of basic functional mobility for frail elderly persons. $J$ Am Geriatr Soc. 1991;39(2):142-148. doi:10.1111/j.1532-5415.1991.tb01616.x

12. Cwikel JG, Fried AV, Biderman A, Galinsky D. Validation of a fall-risk screening test, the Elderly Fall Screening Test (EFST), for community-dwelling elderly. Disabil Rehabil. 1998;20(5):161-167. doi:10.3109/09638289809166077

13. Maidan I, Freedman T, Tzemah R, Giladi N, Mirelman A, Hausdorff JM. Introducing a new definition of a near fall: intra-rater and inter-rater reliability. Gait Posture. 2014;39 (1):645-647. doi:10.1016/j.gaitpost.2013.07.123

14. Arnold CM, Faulkner RA. The history of falls and the association of the timed up and go test to falls and near-falls in older adults with hip osteoarthritis. BMC Geriatr. 2007;7:17. doi:10.1186/1471-2318-7-17

15. Nagai K, Yamada M, Komatsu M, et al. Near falls predict substantial falls in older adults: a prospective cohort study. Geriatr Gerontol Int. 2017;17(10):1477-1480. doi:10.1111/ggi.12898

16. Anagnostis P, Dimopoulou C, Karras S, Lambrinoudaki I, Goulis DG. Sarcopenia in post-menopausal women: is there any role for vitamin D? Maturitas. 2015;82(1):56-64. doi:10.1016/j.maturitas.2015.03.014

17. European Working Group on Sarcopenia in Older People, CruzJentoft AJ, Baeyens JP, Bauer JM, et al. Sarcopenia: european consensus on definition and diagnosis: report of the European Working Group on Sarcopenia in older people. Age Ageing. 2010;39 (4):412-423. doi:10.1093/ageing/afq034.

18. Gale CR, Cooper C, Aihie Sayer A. Prevalence and risk factors for falls in older men and women: the english longitudinal study of ageing. Ageing. 2016;45(6):789-794.

19. Geng Y, Lo JC, Brickner L, Gordon NP. Racial-ethnic differences in fall prevalence among older women: a cross-sectional survey study. BMC Geriatr. 2017;17(1):65. doi:10.1186/s12877-017-0447-y

20. Tricco AC, Thomas SM, Veroniki AA, et al. Comparisons of interventions for preventing falls in older adults: a systematic review and meta-analysis. JAMA. 2017;318(17):1687-1699. doi:10.1001/jama.2017.15006

21. Iolascon G, Moretti A, de Sire A, Calafiore D, Gimigliano F. Effectiveness of calcifediol in improving muscle function in post-menopausal women: a prospective cohort study. Adv Ther. 2017;34(3):744-752. doi:10.1007/s12325-017-0492-0

22. Kellgren JH, Lawrence JS. Radiological assessment of osteo-arthrosis. Ann Rheum Dis. 1957;16(4):494-502. doi:10.1136/ ard.16.4.494

23. Studenski SA, Peters KW, Alley DE, et al. The FNIH sarcopenia project: rationale, study description, conference recommendations, and final estimates. J Gerontol A Biol Sci Med Sci. 2014;69 (5):547-558. doi:10.1093/gerona/glu010 
24. Springer BA, Marin R, Cyhan T, Roberts H, Gill NW. Normative values for the unipedal stance test with eyes open and closed. $J$ Geriatr Phys Ther. 2007;30(1):8-15. doi:10.1519/00139143-200 704000-00003

25. Shimada H, Tiedemann A, Lord SR, et al. Physical factors underlying the association between lower walking performance and falls in older people: a structural equation model. Arch Gerontol Geriatr. 2011;53 (2):131-134. doi:10.1016/j.archger.2010.11.003

26. Veronese N, Bolzetta F, Toffanello ED, et al. Association between short physical performance battery and falls in older people: the Progetto Veneto Anziani Study. Rejuvenation Res. 2014;17 (3):276-284. doi:10.1089/rej.2013.1491

27. O'Hoski S, Bean JF, Ma J, et al. Physical function and frailty for predicting adverse outcomes in older primary care patients. Arch Phys Med Rehabil. 2020;101(4):592-598. doi:10.1016/j.apmr.2019. 11.013

28. Iolascon G, Moretti A, Giamattei MT, Migliaccio S, Gimigliano F. Prevalent fragility fractures as risk factor for skeletal muscle function deficit and dysmobility syndrome in post-menopausal women. Aging Clin Exp Res. 2015;27(Suppl 1):S11-6. doi:10.1007/s40520-0150417-1

29. Hirschfeld HP, Kinsella R, Duque G. Osteosarcopenia: where bone, muscle, and fat collide. Osteoporos Int. 2017;28:2781-2790. doi:10.1007/s00198-017-4151-8

30. Kirk B, Al Saedi A, Duque G. Osteosarcopenia: a case of geroscience. Aging Med (Milton). 2019;2(3):147-156. doi:10.1002/ agm2.12080

31. Kirk B, Zanker J, Duque G. Osteosarcopenia: epidemiology, diagnosis, and treatment-facts and numbers. J Cachexia Sarcopenia Muscle. 2020;11(3):609-618. doi:10.1002/jcsm.12567

32. Bhasin S, Travison TG, Manini TM, et al. Sarcopenia definition: the position statements of the sarcopenia definition and outcomes consortium. J Am Geriatr Soc. 2020. doi:10.1111/jgs. 16372

33. Cruz-Jentoft AJ, Bahat G, Bauer J, et al. Writing Group for the European Working Group on Sarcopenia in Older People 2 (EWGSOP2), and the Extended Group for EWGSOP2. Sarcopenia: revised European consensus on definition and diagnosis. Age Ageing. 2019;48(1):16-31. doi:10.1093/ageing/afy169

34. Abellan van Kan G, Rolland Y, Andrieu S, et al. Gait speed at usual pace as a predictor of adverse outcomes in community dwelling older people an International Academy on Nutrition and Aging (IANA) Task Force. J Nutr Health Aging. 2009;13(10):881-889. doi:10.1007/ s12603-009-0246-Z

35. Studenski S, Perera S, Patel K, et al. Gait speed and survival in older adults. JAMA. 2011;305(1):50-58. doi:10.1001/jama.2010. 1923

36. Peel NM, Kuys SS, Klein K. Gait speed as a measure in geriatric assessment in clinical settings: a systematic review. J Gerontol a Biol Sci Med Sci. 2013;68(1):39-46. doi:10.1093/gerona/gls174

37. Dhalwani NN, Fahami R, Sathanapally H, Seidu S, Davies MJ, Khunti K. Association between polypharmacy and falls in older adults: a longitudinal study from England. BMJ Open. 2017;7(10): e016358. doi:10.1136/bmjopen-2017-016358

38. Johnell K, Jonasdottir Bergman G, Fastbom J, Danielsson B, Borg N, Salmi P. Psychotropic drugs and the risk of fall injuries, hospitalisations and mortality among older adults. Int $J$ Geriatr Psychiatry. 2017;32(4):414-420. doi:10.1002/gps.4483

39. Iolascon G, de Sire A, Calafiore D, Moretti A, Gimigliano R, Gimigliano F. Hypovitaminosis D is associated with a reduction in upper and lower limb muscle strength and physical performance in post-menopausal women: a retrospective study. Aging Clin Exp Res. 2015;27(Suppl 1):S23-30. doi:10.1007/s40520-015-0405-5

40. Iolascon G, Mauro GL, Fiore P, et al. Can vitamin D deficiency influence muscle performance in postmenopausal women? A multicenter retrospective study. Eur $J$ Phys Rehabil Med. 2018;54(5):676-682. doi:10.23736/S1973-9087.17.04533-6
41. Gimigliano F, Moretti A, de Sire A, Calafiore D, Iolascon G. The combination of vitamin D deficiency and overweight affects muscle mass and function in older post-menopausal women. Aging Clin Exp Res. 2018;30(6):625-631. doi:10.1007/s40520-0180921-1

42. Bischoff-Ferrari HA, Dawson-Hughes B, Orav EJ, et al. Monthly high-dose vitamin $\mathrm{D}$ treatment for the prevention of functional decline: a randomized clinical trial. JAMA Intern Med. 2016;176 (2):175-183. doi:10.1001/jamainternmed.2015.7148

43. Li F, Harmer P, Eckstrom E, et al. Cost-effectiveness of a therapeutic tai ji quan fall prevention intervention for older adults at high risk of falling. J Gerontol a Biol Sci Med Sci. 2019;74(9):1504-1510. doi:10.1093/gerona/glz008

44. Centers for Disease Control and Prevention, National Center for Injury Prevention and Control, Division of Unintentional Injury Prevention. Available from: http://www.cdc.gov/homeandrecreatio nalsafety/Falls/steadi/index.html. Accessed June 24, 2020.

45. NICE impact falls and fragility fractures 2018. Available from: https://www.nice.org.uk/Media/Default/About/what-we-do/Intopractice/measuring-uptake/NICE-Impact-falls-and-fragility-fractures. pdf. Accessed June 24, 2020.

46. Perracini MR, Kristensen MT, Cunningham C, Sherrington C. Physiotherapy following fragility fractures. Injury. 2018;49 (8):1413-1417. doi:10.1016/j.injury.2018.06.026

47. Nikander R, Sievänen H, Heinonen A, Daly RM, Uusi-Rasi K, Kannus P. Targeted exercise against osteoporosis: a systematic review and meta-analysis for optimising bone strength throughout life. BMC Med. 2010;8(1):47.

48. Uusi-RasiK R, Karinkanta HS, HeinonenTokola A, Daly RM, UusiRasi K, Kannus P. Targeted exercise against osteoporosis: a systematic review and meta-analysis for optimising bone strength and Fall-Related Fractures in Older Age throughout life. J Osteoporos. 2010;8(1):47.

49. Iolascon G, Di Pietro G, Gimigliano F, et al. Physical exercise and sarcopenia in older people: position paper of the Italian Society of Orthopaedics and Medicine (OrtoMed). Clin Cases Miner Bone Metab. 2014;11(3):215-221.

50. Dent E, Morley JE, Cruz-Jentoft AJ, et al. International Clinical Practice Guidelines for Sarcopenia (ICFSR): screening, diagnosis and management. J Nutr Health Aging. 2018;22(10):1148-1161. doi:10.1007/s12603-018-1139-9

51. Landi F, Calvani R, Tosato M, et al. Protein intake and muscle health in old age: from biological plausibility to clinical evidence. Nutrients. 2016;8(5):E295. doi:10.3390/nu8050295

52. Iolascon G, Gimigliano R, Bianco M, et al. Are dietary supplements and nutraceuticals effective for musculoskeletal health and cognitive function? A scoping review. J Nutr Health Aging. 2017;21 (5):527-538. doi:10.1007/s12603-016-0823-x

53. de Sire A, de Sire R, Petito V, et al. Gut-joint axis: the role of physical exercise on gut microbiota modulation in older people with osteoarthritis. Nutrients. 2020;12(2):574. doi:10.3390/nu12020 574

54. Ballesteros JM, Struijk EA, Rodríguez-Artalejo F, López-García E. Mediterranean diet and risk of falling in community-dwelling older adults. Clin Nutr. 2020;39(1):276-281. doi:10.1016/j.clnu.2019.02. 004

55. Camargo L, Doneda D, de Oliveira VR. Whey protein ingestion in elderly diet and the association with physical, performance and clinical outcomes. Exp Gerontol. 2020;137:110936. doi:10.1016/j. exger.2020.110936

56. Iolascon G, Moretti A, de Sire A, Liguori S, Toro G, Gimigliano F. Pharmacological therapy of sarcopenia: past, present and future. Clin Cases Miner Bone Metab. 2018;15(3):407-415.

57. de Sire A, Invernizzi M, Lippi L, Curci C, Cisari C, Iolascon G. Nutritional supplementation in hip fracture sarcopenic patients: a narrative review. Clin Cases Miner Bone Metab. 2019;16(1):27-30. 
58. Invernizzi M, de Sire A, D'Andrea F, et al. Effects of essential amino acid supplementation and rehabilitation on functioning in hip fracture patients: a pilot randomized controlled trial. Aging Clin Exp Res. 2019;31(10):1517-1524. doi:10.1007/s40520-0181090-y
59. de Sire A, Baricich A, Renò F, Cisari C, Fusco N, Invernizzi M. Myostatin as a potential biomarker to monitor sarcopenia in hip fracture patients undergoing a multidisciplinary rehabilitation and nutritional treatment: a preliminary study. Aging Clin Exp Res. 2020;32(5):959-962. doi:10.1007/s40520-019-01436-8

\section{Publish your work in this journal}

Clinical Interventions in Aging is an international, peer-reviewed journal focusing on evidence-based reports on the value or lack thereof of treatments intended to prevent or delay the onset of maladaptive correlates of aging in human beings. This journal is indexed on PubMed Central, MedLine, CAS, Scopus and the Elsevier
Bibliographic databases. The manuscript management system is completely online and includes a very quick and fair peer-review system, which is all easy to use. Visit http://www.dovepress.com/ testimonials.php to read real quotes from published authors. 